\title{
Convergence of Model Based Shape From Shading
}

\author{
Michael S. Lew Michel Chaudron Nies Huijsmans \\ Computer Science Dept. \\ Leiden University \\ Postbus 9512 \\ 2300 RA Leiden \\ Netherlands \\ email: mlew@cs.leidenuniv.nl
}

\author{
Alfred She Thomas S. Huang \\ Electrical Engineering Dept. \\ University of Illinois \\ Urbana, IL 61801 \\ USA
}

\begin{abstract}
Three dimensional models are used by a wide variety of applications such as face recognition and model based coding for teleconferencing. In face recognition and model based coding the generic 3D model is used to generate images of the individual from any viewpoint. Recent work has been focussed on using shape from shading for refinement of the generic model toward the 3D shape of the individual. However, the previous methods have been based on iteration schemes in which convergence could not be proven. The main goal of this paper is to introduce a provably convergent algorithm for solving the problem of model based shape from shading. Bounds on the number of iterations for attaining error of order $h$ are also given.
\end{abstract}

\section{Introduction}

In a previous paper, we integrated shape from shading techniques with a generic model [Lew, She, and Huang 1995]. However, in the previous work, we had not considered the problem of convergence of the iterative algorithm, nor the time complexity. Thus, the goal of this paper is to introduce a provably convergent algorithm for adjusting the generic model to the individual, and to give bounds on the number of iterations for attaining error of order $h$.

In Section 2 we integrate the generic model with shape from shading and discuss the convergence of the solution. In Section 3, we discuss time complexity and integration of knowledge of discontinuities. In Section 4, conclusions and directions for future work are discussed.

\section{Shape from Shading}

Humans have the ability to estimate shape from one image of a scene. Although it can be shown that the surface shape recovered from the intensity distribution of one image is not unique in general, the ability would still be useful in many applications such as video compression in telecommunications, terrain mapping, robotic navigation, and face recognition. In the past, most work on shape from shading has not proven robust in the presence of noise which occurs in real images. 
In this section, we review the adaptation of standard shape from shading methods to face recognition by the inclusion of relevant constraints of the generic model in the derivation. Furthermore, we prove convergence and uniqueness using a matrix reformulation of the solution.

\subsection{Global Shape from Shading}

First, we consider the case for diffuse cosine shading. These surfaces reflect light as the cosine of the angle between the surface normal and the vector toward the light source as depicted in Figure 1.

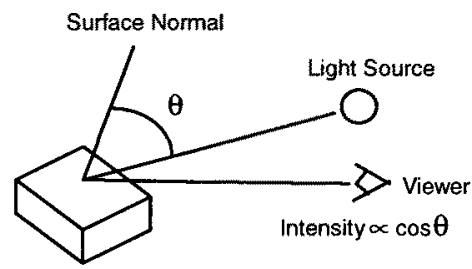

Figure 1. The imaging setup for diffuse cosine shading.

In the literature (i.e. Ballard and Brown [1982]) the diffuse cosine shading is referred to as Lambertian shading. Thus, $R=\mathrm{r}_{0} \cos \theta$. Let $p=\partial f \partial x$ and $q=$ $\partial f / \partial$ for a surface defined by $\mathrm{z}=f(x, y)$, then the Lambertian reflectance, $R_{L}$, can be written as

$$
R_{L}(p, q)=\frac{a p+b q-c}{\sqrt{p^{2}+q^{2}+1}}
$$

where $[a, b, c]$ is the unit vector toward the light source. Horn[1975] solved for the depth map using a characteristic strip method. To reduce the noise sensitivity [Ikeuchi and Horn 1981], a term was added which constrains $p$ and $q$ to be smooth functions. Using variational calculus Euler-Lagrange equations [Courant and Hilbert 1953], we minimize

$$
\varepsilon^{2}=\sum_{x} \sum_{y}[I(x, y)-R(p, q)]^{2} \lambda+\left[p_{x}^{2}+p_{y}^{2}+q_{x}^{2}+q_{y}^{2}\right]
$$

with respect to $p$ and $q$, where $p_{x}=\partial p / \partial x, p_{y}=\partial p / \partial y, q_{x}=\partial q \partial x, q_{y}=\partial q \partial y$, and $\lambda$ is a user set constant, which weighs the contributions from the shading term and the smoothing term.

In order to solve for the unknown surface from the gradient vectors $p$ and $q$, boundary conditions which are usually unknown must be assumed. One commonly applied assumption is that the occluded contours have the same gradient vectors. However, the gradient vectors $p$ and $q$ approach infinity at the occluded contours. This problem can be overcome by using a coordinate system based on the Gaussian sphere [Ikeuchi 1980]. Another solution to the problem of boundary conditions is to impose an additional constraint such as the quadratic surface assumption in local shape from shading. 


\subsection{Method: GLOBAL}

In this section we briefly review our previous method found in Lew, She, and Huang [1995]. We derive a shape from shading method based on an arbitrary reflectance map $R(p, q)$ which can be measured from real images. Specifically, the surface partial derivatives, $p$ and $q$, can be measured from the 3D model using a laser range finder, and the reflected intensity from the real image is proportional to $R(p, q)$. The reflectance map found from the paired 3D model and real image is denoted the generic reflectance map.

In equation $(1), \varepsilon$ can be zero when $I(x, y)$ equals $R(p, q)$ and the gradient vectors are zero, which will be the case when the surface is a plane. Thus, the smoothing term in (1) biases the surface toward a plane. Instead of enforcing gradient smoothness to a plane, we enforce gradient smoothness to the generic model $f^{M}$. Let $p^{M}$ and $q^{M}$ refer to the first partial derivatives of $f^{M}$ with respect to $x$ and $y$. Consequently, the generalized criterion function is

$$
\varepsilon^{2}=\sum_{x} \sum_{y}[I(x, y)-R(p(x, y), q(x, y))]^{2} \lambda+\left[\left(p_{x}-p_{x}^{M}\right)^{2}+\left(p_{y}-p_{y}^{M}\right)^{2}+\left(q_{x}-q_{x}^{M}\right)^{2}+\left(q_{y}-q_{y}^{M}\right)^{2}\right]
$$

The traditional method to determine the minimum of equation (2) is to apply variational calculus to derive the necessary conditions for a minimum. Following such an approach and creating an iterative scheme using the Gauss-Seidel method gives

$$
\begin{aligned}
& p_{t+1}(i, j)=\tilde{p}_{t}(i, j)+p^{M}(i, j)-\tilde{p}^{M}(i, j)+\frac{\lambda}{4}\left(I(i, j)-R\left[p_{t}(i, j), q_{t}(i, j)\right]\right) \frac{\partial R}{\partial p}\left[p_{t}(i, j), q_{t}(i, j)\right] \\
& q_{t+1}(i, j)=\tilde{q}_{t}(i, j)+q^{M}(i, j)-\tilde{q}^{M}(i, j)+\frac{\lambda}{4}\left(I(i, j)-R\left[p_{t}(i, j), q_{t}(i, j)\right]\right) \frac{\partial R}{\partial q}\left[p_{t}(i, j), q_{t}(i, j)\right]
\end{aligned}
$$

where

$$
\begin{aligned}
& \tilde{p}_{t}(i, j)=\frac{p_{t}(i-1, j)+p_{t}(i+1, j)+p_{t}(i, j-1)+p_{t}(i, j+1)}{4} \\
& \tilde{p}^{M}(i, j)=\frac{p^{M}(i-1, j)+p^{M}(i+1, j)+p^{M}(i, j-1)+p^{M}(i, j+1)}{4}
\end{aligned}
$$

and

$$
\begin{aligned}
& \tilde{q}_{t}(i, j)=\frac{q_{t}(i-1, j)+q_{t}(i+1, j)+q_{t}(i, j-1)+q_{t}(i, j+1)}{4} \\
& \tilde{q}^{M}(i, j)=\frac{q^{M}(i-1, j)+q^{M}(i+1, j)+q^{M}(i, j-1)+q^{M}(i, j+1)}{4}
\end{aligned}
$$




\subsection{Existence and Convergence}

Two interesting aspects of an iterative solution are existence and convergence. If $R(p, q)$ is continuous then there exists an $\mathbf{x}$ which satisfies equation (2) [Lee 1989]. One method to prove convergence is to show that

$$
\frac{\left\|\mathbf{x}_{\mathbf{t}}-\mathbf{x}^{*}\right\|}{\left\|\mathbf{x}_{\mathrm{t}-1}-\mathbf{x}^{*}\right\|}<\alpha<1
$$

where $\mathbf{x}_{\mathbf{t}}$ is the estimate of the solution at iteration $t$, and $\mathbf{x}^{*}$ is the correct solution, and $\alpha$ is a fixed positive constant. We can rewrite (5) as $\left\|\mathbf{x}_{\mathbf{t}}-\mathbf{x}^{*}\right\|<\alpha^{\mathrm{t}}\left\|\mathbf{x}_{0}-\mathbf{x}^{*}\right\|$ or as $\left\|\mathbf{x}_{\mathbf{t}}-\mathbf{x}^{*}\right\|<\varepsilon$, and for $\alpha<1, \lim _{\mathrm{t} \rightarrow \infty} \alpha^{\mathrm{t}}=0$ and $\lim _{\mathrm{t} \rightarrow \infty} \mathbf{x}_{\mathrm{t}}=\mathbf{x}^{*}$

First, we formulate the solution to the criterion function in a manner similar to Lee [1989]. Let $S$ denote the region of interest. Rewriting equations (3) and (4) in matrix form and accounting for boundary conditions yields

$$
\mathbf{M}\left(\mathbf{x}_{t}-\mathbf{x}^{\mathrm{M}}\right)=-\lambda \mathbf{h}^{2} \mathbf{b}\left(\mathbf{x}_{t}\right)+\mathbf{r}
$$

where

$\lambda$ is the weighting constant between the shading term and the smoothing term.

$\mathrm{h}$ is the mesh size $\mathbf{M}^{-1}=\operatorname{diag}\left(\mathbf{A}^{-1}, \mathbf{A}^{-1}\right)$

$\mathbf{A}$ is the Laplacian Matrix [Isaacson \& Keller 1966]

$$
\mathbf{A}=\left[\begin{array}{cccc}
B & -I & \cdot & \cdot \\
-I & B & \ddots & \cdot \\
\cdot & \ddots & \ddots & -I \\
\cdot & \cdot & -I & B
\end{array}\right] \quad \mathbf{B}=\left[\begin{array}{cccc}
4 & -1 & \cdot & \cdot \\
-1 & 4 & \ddots & \cdot \\
\cdot & \ddots & \ddots & -1 \\
\cdot & \cdot & -1 & 4
\end{array}\right]
$$

Let $\mathrm{N}=\mathrm{nm}=$ total number of grid points.

$A$ is $\mathbf{N} \times \mathbf{N}, B$ is $n \times n$ tridiagonal matrix and $I$ is an $n \times n$ identity matrix

$$
\begin{aligned}
& \mathbf{x}_{\mathrm{t}}=\left(\ldots, \mathrm{p}_{\mathrm{t}}(\mathrm{i}, \mathrm{j}), \ldots, \ldots, \mathrm{q}_{\mathrm{t}}(\mathrm{i}, \mathrm{j}), \ldots\right)^{\mathrm{T}} \\
& \mathbf{x}^{\mathrm{M}}=\left(\ldots, \mathrm{p}^{\mathrm{M}}(\mathrm{i}, \mathrm{j}), \ldots, \ldots, \mathrm{q}^{\mathrm{M}}(\mathrm{i}, \mathrm{j}), \ldots\right)^{\mathrm{T}} \\
& \mathbf{r}=(\ldots, \mathrm{r}(\mathrm{i}, \mathrm{j}), \ldots)^{\mathrm{T}} \text { boundary point constants which satisfy }(6) \text { at the } \\
& \text { noninterior points. } \\
& \mathbf{b}\left(\mathbf{x}_{\mathrm{t}}\right)=\left(\ldots,\left(\mathrm{R}\left(\mathrm{p}_{\mathrm{t}}, \mathrm{q}_{\mathrm{t}}\right)-\mathrm{I}(\mathrm{i}, \mathrm{j})\right)\left(\partial \mathrm{R}\left(\mathrm{p}_{\mathrm{t}}, \mathrm{q}_{\mathrm{t}}\right) / \partial \mathrm{p}\right), \ldots, \ldots,\left(\mathrm{R}\left(\mathrm{p}_{\mathrm{t}}, \mathrm{q}_{\mathrm{t}}\right)\right.\right. \\
& \left.\mathrm{I}(\mathrm{i}, \mathrm{j}))\left(\partial \mathrm{R}\left(\mathrm{p}_{\mathrm{t}}, \mathrm{q}_{\mathrm{t}}\right) / \partial \mathrm{q}\right), \ldots\right)^{\mathrm{T}}
\end{aligned}
$$

Since $\mathbf{M}$ is positive definite and symmetric, it has an inverse. Multiplying by $\mathbf{M}^{-1}$ and solving for $\mathbf{x}$ yields

$$
\mathbf{x}_{\mathrm{t}}=-\lambda \mathrm{h}^{2} \mathbf{M}^{-1} \mathbf{b}\left(\mathbf{x}_{\mathrm{t}-1}\right)+\mathbf{M}^{-1} \mathbf{r}+\mathbf{x}^{\mathrm{M}}
$$

We denote the solution to equation (7) as $\mathbf{x}^{*}$ where

$$
\mathbf{x}^{*}=-\lambda h^{2} \mathbf{M}^{-1} \mathbf{b}\left(\mathbf{x}^{*}\right)+\mathbf{M}^{-1} \mathbf{r}+\mathbf{x}^{\mathrm{M}}
$$


We assume that $\left(\mathrm{R}\left(\mathrm{p}_{\mathrm{t}}, \mathrm{q}_{\mathrm{t}}\right)-\mathrm{I}(\mathrm{i}, \mathrm{j})\right)\left(\partial \mathrm{R}\left(\mathrm{p}_{\mathrm{t}}, \mathrm{q}_{\mathrm{t}}\right) / \partial \mathrm{p}\right)$ and $\left(\mathrm{R}\left(\mathrm{p}_{\mathrm{t}}, \mathrm{q}_{\mathrm{t}}\right)-\mathrm{I}(\mathrm{i}, \mathrm{j})\right)\left(\partial \mathrm{R}\left(\mathrm{p}_{\mathrm{t}}, \mathrm{q}_{\mathrm{t}}\right) / \partial \mathrm{q}\right)$ are Lipschitz functions for all $(\mathrm{i}, \mathrm{j})$. This results in

$$
\left\|\mathbf{b}(\mathbf{x})-\mathbf{b}\left(\mathbf{x}^{\prime}\right)\right\| \leq v\left\|\mathbf{x}-\mathbf{x}^{\prime}\right\|
$$

where $v$ is the maximum Lipschitz constant of $\left(\mathrm{R}\left(\mathrm{p}_{\mathrm{t}}, \mathrm{q}_{\mathrm{t}}\right)-\mathrm{I}(\mathrm{i}, \mathrm{j})\right)\left(\partial \mathrm{R}\left(\mathrm{p}_{\mathrm{t}}, \mathrm{q}_{\mathrm{t}}\right) / \partial \mathrm{p}\right)$ and $\left(R\left(p_{t}, q_{t}\right)-I(i, j)\right)\left(\partial R\left(p_{t}, q_{t}\right) / \partial q\right)$ for all $(i, j)$.

From Isaacson and Keller[1966] and Lee [1989],

$$
\begin{gathered}
\left\|\mathbf{M}^{-1}\right\|=\left\|\mathbf{A}^{-1}\right\| \leq \frac{1}{4 s(n, m)} \\
\text { where } s(n, m)=\sin ^{2} \frac{\pi}{2(m+1)}+\sin ^{2} \frac{\pi}{2(n+1)} \\
\mathrm{m}=\text { number of rows in } S \\
\mathrm{n}=\text { maximum number of grid points in a single row in } S
\end{gathered}
$$

Then subtracting (8) from (7) gives

$$
\mathbf{x}_{t}-\mathbf{x}^{*}=-\lambda \mathbf{h}^{2} \mathbf{M}^{-1}\left(\mathbf{b}\left(\mathbf{x}_{t-1}\right)-\mathbf{b}\left(\mathbf{x}^{*}\right)\right)
$$

Using the Cauchy-Schwarz Inequality gives

$$
\frac{\left\|\mathbf{x}_{\mathbf{t}}-\mathbf{x}^{*}\right\|}{\left\|\mathbf{b}\left(\mathbf{x}_{\mathbf{t}}\right)-\mathbf{b}\left(\mathbf{x}^{*}\right)\right\|} \leq \lambda h^{2}\left\|\mathbf{M}^{-1}\right\|
$$

Substituting equations (9) and (10) into (12) gives

$$
\frac{\left\|\mathbf{x}_{\mathrm{t}}-\mathbf{x}^{*}\right\|}{\left\|\mathbf{x}_{\mathrm{t}-1}-\mathbf{x}^{*}\right\|} \leq \frac{\lambda h^{2} v}{4 s(n, m)}
$$

If $\lambda \in\left[0, \frac{4 s(n . m)}{h^{2} v}\right)$ then $\frac{\left\|\mathbf{x}_{1}-\mathbf{x}^{2}\right\|}{\left\|\mathbf{x}_{t-1}-\mathbf{x}^{4}\right\|}<\alpha<1$,

and $\mathbf{x}_{\mathbf{t}}$ converges to $\mathbf{x}^{*}$.

\section{Issues}

In the previous section, we proved that for a certain range of lambda, the algorithm would converge. In this section we discuss the time complexity to attain error of order $\mathrm{g}$, and we comment on inclusion of discontinuity information into the objective criterion function in equation (2).

\subsection{Accuracy and Computational Complexity}

Let $\alpha=\frac{\lambda h^{2} v}{4 s(n, m)}<1$, then from equation (13), the error of $\mathbf{x}_{t+1}$ is of order $\alpha^{t}$. Thus, an approximation to the solution can be constructed of order $g$ in $O(\log (1 / g))$ iterations.Furthermore for a rectangular region of size $\mathrm{N}$, the total cost of constructing an estimate with error of order $\mathrm{g}$ is $\mathrm{O}(N \log N \log (1 / \mathrm{g}))$ using a Fast Fourier Transform approach [Lee 1989]. 


\subsection{Discontinuities}

In many applications, apriori knowledge about discontinuities in the depth map is given. One method of modeling discontinuities in the criterion function is to alter $\lambda$ from a constant to a function such as $\lambda(x, y)$. When $\lambda$ is large then the contribution of the smoothing term will be negligible which allows for a closer modeling of discontinuities. It is important to note that the condition on $\lambda$ in (14) becomes a condition on the maximum of $\lambda(x, y)$ over all $(x, y)$. Thus, convergence is not guaranteed for a discontinuous depth map. Convergence is guaranteed when the minimum and maximum $\lambda(\mathrm{x}, \mathrm{y})$ over all $(\mathrm{x}, \mathrm{y})$ satisfies (14).

\section{Conclusions}

This paper presented theoretical sufficient conditions for convergence of the shape from shading algorithm of Lew, She, and Huang[1995]. The criterion function integrating the generic model was solved using the traditional variational calculus method and using the matrix formulation. The advantage to the matrix formulation is that sufficient conditions for convergence can be proved analytically. Furthermore, accuracy, computational complexity and incorporation of depth discontinuites were discussed. Regarding future work, it would be interesting to pursue the incorporation of discontinuities into the algorithm

\section{References}

Ballard, D. and C. M. Brown, Computer Vision, Prentice-Hall, Englewood Cliffs, New Jersey, 1982.

Courant, R. and D. Hilbert, Methods of Mathematical Physics, vol. 1, Interscience Publishers, New York, 1953.

Horn, B. K. P., "Obtaining Shape from Shading Information," Psychology of Machine Vision, McGrawHill, New York, pp. 115-155, 1975.

Ikeuchi, K., "Shape from Regular Patterns - An Example of Constraint Propagation in Vision," Proceedings of the Fifth International Joint Conference on Pattern Recognition, Miami Beach, Florida, 1980.

Ikeuchi, K., and B. K. P. Horn, "Numerical Shape from Shading and Occluding Boundaries," Artificial Intelligence, vol. 17, pp. 141-184, 1981.

Isaacson, E., and H. B. Keller, Analysis of Numerical Methods, John Wiley \& Sons, New York, 1966.

Lee, David, "A Provable Convergent Algorithm for Shape From Shading," Shape From Shading edited by Horn and Brooks, MIT Press, Cambridge, pp. 349-373.

Lew, M., A. C. She and T. S. Huang, "Intermediate Views For Face Recognition," Lecture Notes in Computer Science: Proceedings of the 6th International Conference on Computer Analysis of Images and Patterns, pp. 138-145, 1995. 